\title{
Incidence of subsequent primary cancers and radiation-induced subsequent primary cancers after low dose-rate brachytherapy monotherapy for prostate cancer in long-term follow-up
}

Kristiina Vuolukka ( $\nabla$ kristiina.vuolukka@kuh.fi )

Kuopio University Hospital https://orcid.org/0000-0001-9067-0566

\section{Päivi Auvinen}

Kuopion yliopistollinen sairaala

Jan-Erik Palmgren

Kuopion yliopistollinen sairaala

\section{Sirpa Aaltomaa}

Kuopion yliopistollinen sairaala

\section{Vesa Kataja}

Keski-Suomen keskussairaala

\section{Research article}

Keywords: Prostate cancer, low dose-rate brachytherapy, subsequent primary cancer, radiation induced subsequent primary cancer

Posted Date: November 19th, 2019

DOI: https://doi.org/10.21203/rs.2.17489/v1

License: (c) (i) This work is licensed under a Creative Commons Attribution 4.0 International License. Read Full License

Version of Record: A version of this preprint was published at BMC Cancer on May 20th, 2020. See the published version at https://doi.org/10.1186/s12885-020-06960-9. 


\section{Abstract}

Background As aging is the most significant risk factor for cancer development, long-term prostate cancer (PCa) survivors have an evident risk of developing subsequent primary cancers (SPCs).

Radiotherapy itself is an additional risk factor for cancer development and the SPCs appearing beyond five years after radiotherapy in the original treatment field can be considered as radiation-induced subsequent primary cancers (RISPCs).Material and Methods During the years 1999-2008, 241 patients with localized PCa who underwent LDR-BT with I125 and were followed-up in Kuopio University Hospital, were included in this study. In this study the incidences and types of SPCs and RISPCs with a very long follow-up time after low dose-rate brachytherapy (LDR-BT) were evaluated.Results During the median follow-up time of 11.4 years, a total of $34(14.1 \%)$ patients developed a metachronous SPC. The crude incidence rate of RISPC was $1.7 \%(n=4)$. Half of the SPC cases $(50 \%)$ were diagnosed during the latter half of the follow-up time as the risk to develop an SPC continued throughout the whole follow-up time with the actuarial 10-year SPC rate of 7.0\%. The crude death rates due to metachronous out-of-field SPCs and RISPCs were high, $50 \%$ and $50 \%$, respectively.Conclusion The crude rate of SPC was significant, but in line with previous data. The incidence of RISPC was very low supporting the role of LDR-BT as a safe treatment option for patients with localized PCa. However, both the SPCs and the RISPCs after LDR-BT were serious diseases with high mortality rates.

\section{Background}

With over 1.2 million new cases diagnosed worldwide annually, prostate cancer (PCa) is the second most common solid tumor in males (1). As aging is the most significant risk factor for cancer development, long-term PCa survivors have, per se, an evident risk of developing multiple subsequent primary cancers (SPCs). Radiotherapy (RT) is also a risk factor for cancer development and SPCs appearing beyond five years after RT in the original treatment field can be considered as radiation-induced subsequent primary cancers (RISPCs) $(2,3)$. There is also an increased risk of SPCs outside the radiation field either because of radiation scattering or from radiation-induced genetic alterations without direct in-field exposure. The risks of SPC and RISPC become even more relevant as the median overall survival (OS) of the RT treated patients with localized PCa now exceeds 12 years (4). Thus, not only the efficacy and the adverse event profile of the treatment approach, but also the potential risk of SPC and RISPC may influence the selection of the treatment modality for PCa.

Several treatment options are available for localized PCa, yet no consensus regarding the optimum primary treatment exists $(5,6)$. The RT modality may have an effect on the incidence of both SPCs and RISPCs as this is well supported with data from risk analyses of PCa patients treated with different RT modalities (3). Most of the studies have included patients treated with external beam radiotherapy (EBRT) in varying planned target volumes, fractionation and techniques, while some have included patients treated with EBRT in combination with either low dose-rate brachytherapy (LDR-BT) or high dose-rate 
boosts. The extensive systematic reviews conducted by Murray et al. and Wallis et al. revealed a small increased risk of SPC in irradiated PCa patients with the risk of RISPC being estimated to be small but increasing with time $(2,3)$. Since they have very heterogenic cohorts with varying follow-up times, these results are difficult to interpret and it is not possible to draw definitive conclusions regarding different RT modalities and the actual risks of SPCs and RISPCs.

Considering the irradiated volume, it could be expected that the SPC risk after LDR-BT would be lower than the corresponding risk after EBRT due to the lack of scattering radiation and the low risk of radiation leakage. The International Commission on Radiological Protection has made this conclusion already in 2005 (7). Abdel-Wahab et al. have confirmed this finding, as significantly fewer SPCs were diagnosed among patients treated with brachytherapy as compared to EBRT, $4.7 \%$, and $10.3 \%$ respectively, in the SEER tumor registry data from the years 1988-2002 (8).

Despite the increasing role of moderate and ultra-hypofractionated EBRT and stereotactic body radiotherapy (SBRT) techniques in the management of localised PCa, LDR-BT is still a valid treatment modality for low and favourable profile (Gleason score $3+4$ ) intermediate-risk patients and is offered in several centers around the world. LDR-BT enables a single session treatment with very high total doses (145-150 Gy) to the target and steep dose gradients to the organs at risk surrounding the prostate. With technical advances in imaging and seed implantation, the LDR-BT in treating localized PCa has gained further popularity.

In Kuopio University Hospital (KUH), LDR-BT for patients with localized low and intermediate-risk PCa was initiated in 1999. The long-term efficacy and toxicity results of LDR-BT in this patient cohort has been published recently (9). The aim of this study was to analyze the overall SPC rate and the incidence of potential RISPCs among Nordic patients with localized PCa with an 8-16 years follow-up time.

\section{Methods}

During the years 1999-2008, 241 patients from KUH district with localized PCa were treated with LDR-BT monotherapy with $\mathrm{I}^{125}$ seeds according to ESTRO/EAU/EORTC guidelines (10). All relevant patient data was scrutinized to identify subsequent cancer diagnoses (excluding basal cell carcinoma) evolving a minimum of 12 months after LDR-BT, thus fulfilling the criteria of a metachronous SPC. For the purposes of this study, the SPCs were categorised as in-field (IF) malignancies including all SPCs arising within the true pelvis (i.e. in the prostate, the bladder, the rectum or the anus) and as out-of-field (OOF) cancers including all extra pelvic SPCs. The time to the SPC and RISPC was defined as the time from the date of 
LDR-BT to the date of the clinico-pathological diagnosis of SPC and RISPC, respectively. The statistical analyses were performed with SPSS Statistics 22 software (SPSS, Chicago, IL).

\section{Results}

The patient demographics and clinical data on the PCas are shown in Table 1. During the follow-up time (median follow-up (mFU) of 11.4 years, range 8-16 years), a total of 34 patients developed a metachronous subsequent primary cancer, the crude incidence rate of SPC thus being $14.1 \%$. Two of the patients developed also another subsequent cancer. The most abundant SPCs were lung and colorectal cancers both diagnosed in six patients and each representing $16.7 \%$ of all the SPCs (Table 2). The crude incidence rate of RISPC was $1.7 \%$ as four SPCs fulfilled the criteria for RISPC (Tables 2 and 3).

As shown in Figure 1, every second (17 cases, 50\%) of the SPCs was diagnosed during the first 7.6 years of the follow-up and the risk to develop an SPC continued throughout the follow-up time. The actuarial 10-year SPC rate was 7.0\%. Twenty-three of the SPCs developed more than five years after LDR-BT, but only four of these developed in the IF area (Table 2). The median times to develop a metachronous OOF SPC and an RISPC were 7.0 years (range, 1.5-12.6 years) and 9.8 years (range, 9.3-13.3 years) after LDR$B T$, respectively. The third primary cancers, a squamous cell carcinoma of the vocal cords and an adenocarcinoma of the hepatic flexure of the colon, were diagnosed at 12.6 and 12.8 years after LDR-BT, respectively.

At the mFU of 11.4 years, the OS rates for all patients $(n=241)$, patients with no metachronous SPC $(n=207)$, patients with a metachronous OOF SPC $(n=30)$ and patients with an RISPC $(n=4)$ were $66.4 \%$, $69.1 \%, 50 \%$ and $50 \%$, respectively. Out of the 81 deaths during the follow-up, 53 (65.4\%) were due to comorbidities or trauma and $11(13.6 \%)$ due to PCa. Seventeen (21.0\%) patients died due to a malignancy other than PCa: 15 due to a metachronous OOF SPC and two due to an RISPC. The actuarial 10-year death-rates due to PCa, metachronous OOF SPC and RISPC were $3.0 \%, 3.0 \%$ and $<1.0 \%$, respectively.

\section{Discussion}

In this real-life cohort of 241 PCa patients with a median age of 65 and an mFU of 11.4 years after LDRBT monotherapy, 34 (14.1\%) patients were diagnosed with an SPC. The incidence is low, but clinically significant, since every second patient diagnosed with an SPC died from this malignancy. The actuarial 10-year death-rate due to an SPC was equal to the actuarial 10-year death-rate due to PCa itself. The majority of the SPCs were diagnosed during the latter half of the follow-up period emphasizing the significance of a long follow-up time. 
In European studies, the values of 10-year cumulative incidence of any SPC are concordant with our results $(11,12)$. A study from Australia, a country with a relatively higher incidence of melanoma, reported a 10-year cumulative SPC incidence of $18.5 \%$ (13). However, with the exception of the study by Cosset et al. from France, little is known about the SPC incidence in cohorts with mFU times extending over 10 years after LDR-BT monotherapy (14). With 675 patients treated with LDR-BT and the mFU of 11.0 years, the 10 -year cumulative incidence of secondary malignancies was $7 \%$, similar to our results (14).

Compared to the general population, a significantly increased risk of bladder cancer after LDR-BT has been reported in single-centre cohorts from the Netherlands, the UK and Australia, but only during the first four to five years after LDR-BT and among patients under 60 years of age (11-13). This might be due to increased urological surveillance after the treatment $(11,13)$, as the incidences of bladder cancer were higher than expected also among RP patients, the lead-time or screening bias may explain these results (12). However, the influence of radiotherapy as a possible risk factor for a second primary cancer cannot be excluded.

In European single-centre LDR-BT-cohorts, no increased risk of rectal cancer has been observed $(11,12,14)$. Hamilton et al. did not find any increased risk of pelvic SPCs among PCa patients treated with BT as compared to those treated with RP (15) and Cosset et al. confirmed a very low risk, if any, of RISPC after LDR-BT (14). In our cohort, the cumulative incidence of RISCP was very low, since only four patients developed an RISPC. The actuarial 10-year death-rate due to RISPC was $<1 \%$. In SEER based analysis by Abdel-Wahab, there were no significant differences found in the incidence of RISPCs between different RT-groups (EBRT, BT or their combination) showing that LDR-BT is as safe as other RT treatments (8).

In addition to aging and effects related to the treatment, radiation in this context, SPCs can arise because of a genetic predisposition and a shared etiological background. Furthermore, lifestyle factors, i.e. smoking habits, and workplace exposure to carcinogenic chemicals may contribute to the SPC incidence, also in organs at risk. Patients may also have synchronous malignancies and sometimes it is difficult to determine which is the primary and which is the subsequent primary cancer. In our cohort, six $(2.5 \%)$ patients had a medical history of previous cancer, which categorizes the PCa itself to the position of a subsequent primary cancer.

As the nature of the low-risk and favorable-profile intermediate-risk PCa is non-aggressive and the efficacy of LDR-BT for this particular malignancy is high, patients do survive and live long enough for some of them to develop another malignancy. SPCs seem to be deadly diseases, irrespective of the location as in a cohort of 2418 BT-treated men with mFU of 5.8 years, $41 \%$ of the patients developing an 
SPC died due it (15). In our study with mFU almost twice as long, of the patients diagnosed with an OOF SPC, $50 \%$ (15 patients out of the 30 patients with an OOF SPC) died due it. As the absolute numbers of SPCs are low, the clinical burden is not overwhelming. On the other hand, to our surprise, the risk of dying due to a metachronous SPC was equal with the risk of dying due to the primary cancer, PCa itself at 10 years after LDR-BT, because the prognosis of PCa patients is very good. Equally, with RISPCs, in our cohort the numbers are very small, but nonetheless the $50 \%$ death-rate due to the RISPCs is noteworthy.

In our cohort, every second metachronous SPC was diagnosed during the first 7.6 years. The risk to develop an SPC continued steadily, if not even more steeply, over the follow-up period, as shown in Figure 1 , and the same phenomenon has been reported previously in studies with European and Western patient populations $(16,17)$. As the OS of PCa patients is lengthening and the aging is a major risk factor for cancer development in general, this observation is not unexpected. At the cut-off point, the majority of the patients ( 160 out of $241,66.4 \%$ ) in our cohort were still alive and could possible develop an SPC or an RISPC in the future. Only a very long-term follow-up after RT will reveal the life-long risk of SPCs and RISPC among the curatively treated cancer patients.

\section{Conclusions}

With an mFU of 11.4 years in our real-life PCa patient cohort treated with LDR-BT, the follow-up time is sufficient to analyze the risk of SPCs and also the risk of RISPCs. The crude rate of SPC was significant, but in line with previous data and of the same order of magnitude, as the cancer incidence among a similar aged general Finnish male population (18), supporting the safety of LDR-BT as a treatment option for patients with localized PCa. LDR-BT for localized PCa resulted in a very low number of RISPCs, altough it is noteworthy, that both the SPCs and the RISPCs are serious diseases with high mortality rates emphasizing the significance of a long follow-up time of the patients.

\section{Abbreviations}

EBRT External beam radiotherapy

IF In-field

KUH Kuopio University Hospital

LDR-BT Low dose-rate brachytherapy

mFU Median follow-up

OOF Out-of-field

OS Overall survival 
PCa Prostate cancer

RISPC Radiation-induced subsequent primary cancer

RT Radiotherapy

SBRT Stereotactic body radiotherapy

SPC Subsequent primary cancer

\section{Declarations}

\section{Ethics approval and consent to participate}

This study has been approved by the ethics committee of the Northern Savo Hospital District with the reference number of 73/13.02.00/2015.

\section{Consent for publication}

An informed consent has been obtained from each patient before the treatment. The data within the manuscript is entirely unidentifiable, thus no consent for publication is required.

Availability of data and materials

The data that support the findings of this study are available from Kuopio University Hospital but restrictions apply to the availability of these data, which were used under license for the current study, and so are not publicly available. Data are however available from the authors upon reasonable request and with permission of Kuopio University Hospital.

\section{Competing interests}

The authors declare that they have no competing interests.

\section{Funding}

The corresponding author has received financial support from Instrumentarium Science Foundation and Finnish Society of Oncology in the form of an unrestricted research grant.

\section{Authors' contributions}

$\mathrm{KV}$ gathered, analyzed and interpreted the patient data regarding the subsequent primary cancers and the radiation-induced subsequent primary cancers. KV was a major contributor in writing the manuscript. PA participated in the interpretation of the results and contributed in the writing of the manuscript. JEP participated in analyzing the data and SA and VK have drafted the work and revised the manuscript. All authors have read and approved the final manuscript. 
Acknowledgements

The authors acknowledge biostatistician Tuomas Selander for graphical expertise.

\section{References}

[1] World Health Organization. WHO Cancer Today.

http://gco.iarc.fr/today/data/factsheets/populations/900-world-fact-sheets.pdf. Accessed Nov 7th, 2019.

[2] Murray L, Henry A, Hoskin P, Siebert F-A, Venselaar Jack. Second primary cancers after radiation for prostate cancer: a systematic review of the clinical data and impact of treatment technique. Radiother Oncol. 2014;110(2):213-28.

[3] Wallis CJ, Mahar AL, Choo R, Herchorn S, Kodama RT, Shah PS et al. Second malignancies after radiotherapy for prostate cancer: systematic review and meta-analysis. BMJ. 2016;352:i851.

[4] Barbosa PV, Thomas IC, Srinivas S, Buyyounouski MK, Chung BI, Chertow GM et al. Overall survival in patients with localized prostate cancer in the US veterans health administration: Is PIVOT generalizable? Eur Urol. 2016;70(2):227-30.

[5] Parker C, Gillessen S, Heidenreich A, Horwich A. Cancer of the prostate: ESMO clinical practice guidelines for diagnosis, treatment and follow-up. Ann Oncol. 2015;26 Suppl 5:v69-77.

[6] Mottet N, van den Bergh RCN, Briers E, Cornford P, De Santis M, Fanti S et al. EAU Guidelines: Prostate Cancer. https://uroweb.org/guideline/prostate-cancer/. Accessed Nov $7^{\text {th }} 2019$.

[7] International Commission on Radiological Protection. Radiation safety aspects of brachytherapy for prostate cancer using permanently implanted sources. A report of ICRP publication 98. Ann ICRP. 2005;35(3):3-50.

[8] Abdel-Wahab M, Reis IM, Hamilton K. Second primary cancer after radiotherapy for prostate cancer--a seer analysis of brachytherapy versus external beam radiotherapy. Int $\mathrm{J}$ Radiat Oncol Biol Phys. 2008;72(1):58-68.

[9] Vuolukka K, Auvinen P, Palmgren JE, Voutilainen T, Aaltomaa S, Kataja V. Long-term efficacy and urological toxicity of low-dose-rate brachytherapy (LDR-BT) as monotherapy in localized prostate cancer. Brachytherapy. 2019;18(5)583-588.

[10] Ash D, Flynn A, Battermann J, de Reijke T, Lavagnini P, Blank L. ESTRO/EAU/EORTC recommendations on permanent seed implantation for localized prostate cancer. Radiother Oncol. 2000;57(3):315-21.

[11] Musunuru H, Mason M, Murray L, Al-Qaisieh B, Bownes P, Smith J et al. Second primary cancers occurring after I-125 brachytherapy as monotherapy for early prostate cancer. Clin Oncol (R Coll Radiol). 
2014;26(4):210-5.

[12] Hinnen KA, Schaapveld M, van Vulpen M, Battermann JJ, van der Poel H, van Oort IM et al. Prostate brachytherapy and second primary cancer risk: a competitive risk analysis. J Clin Oncol. 2011;29(34):4510-5.

[13] Fernandez Ots A, Browne L, Chin YS, Malouf D, Wong K, Bucci J. The risk of second malignancies after 125 I prostate brachytherapy as monotherapy in a single Australian institution. Brachytherapy. 2016;15(6):752-759.

[14] Cosset JM, Belin L, Wakil G, Flam T, Thiounn N, Pierrat N et al. Second malignancies after permanent implant prostate cancer brachytherapy: A single-institution study of 675 patients treated between 1999 and 2003. Cancer Radiother. 2017;21(3):210-215.

[15] Hamilton SN, Tyldesley S, Hamm J, Jiang WN, Keyes M, Pickles T et al. Incidence of second malignancies in prostate cancer patients treated with low-dose-rate brachytherapy and radical prostatectomy. Int J Radiat Oncol Biol Phys. 2014;90(4):934-941.

[16] Van Hemelrijck M, Feller A, Garmo H, Valeri F, Korol D, Dehler S et al. Incidence of second malignancies for prostate cancer. PLoS One. 2014;9(7):e102596.

[17] Jin T, Song T, Deng S, Wang K. Radiation-induced secondary malignancy in prostate cancer: a systematic review and meta-analysis. Urol Int. 2014;93(3):279-88.

[18] The NORDCAN project - Cancer statistics from the Nordic countries. http://wwwdep.iarc.fr/NORDCAN/english/frame.asp. Accessed Nov $8^{\text {th }} 2019$.

\section{Tables}

Table 1. Patient demographics. 


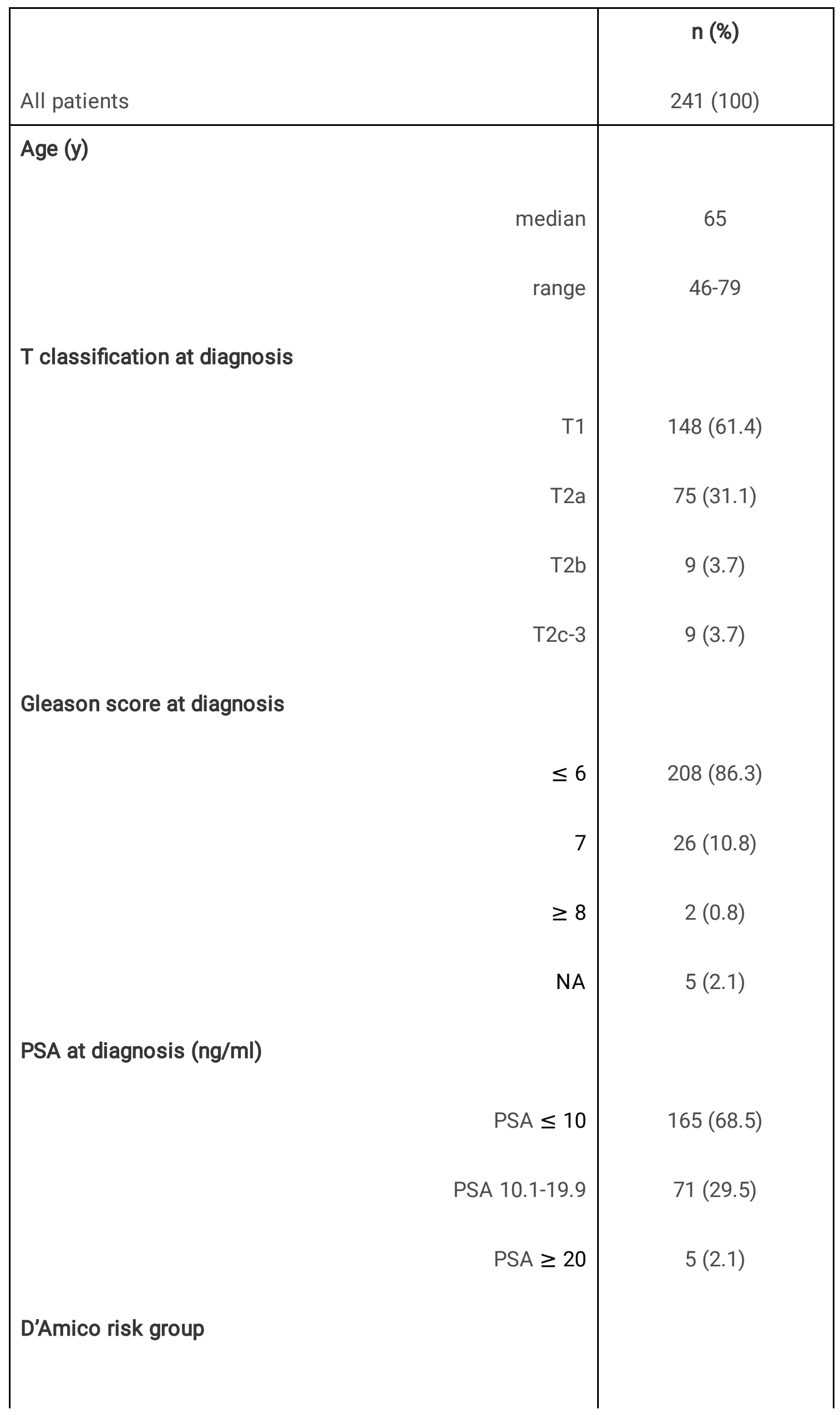




\begin{tabular}{|l|r|r|} 
low & $142(58.9)$ \\
intermediate & high & $135.3)$ \\
Short term ADT & no & \\
PSA, prostate-specific antigen; ADT, androgen deprivation therapy; NA, not available \\
\hline
\end{tabular}

Table 2. Metachronous SPCs, time to SPCs and locations of the SPCs after LDR-BT. 


\begin{tabular}{|c|c|c|c|c|c|c|}
\hline SPCs, organs involved & $\mathbf{n}$ & $\%$ & $<5$ years & $\geq 5$ years & OOF & IF \\
\hline All & 36 & 100 & 13 & 23 & 32 & 4 \\
\hline Lung & 6 & 16,7 & 2 & 4 & 6 & \\
\hline Colon & 5 & 13,9 & 2 & 3 & 5 & \\
\hline Skint & 5 & 13,9 & 3 & 2 & 5 & \\
\hline Hematologic & 4 & 11,1 & 3 & 1 & 4 & \\
\hline Bladder & 2 & 5,6 & & 2 & & 2 \\
\hline Rectum & 1 & 2,8 & & 1 & & 1 \\
\hline Prostate (SCC) & 1 & 2.8 & & 1 & & 1 \\
\hline Othert+ & 12 & 33.3 & 3 & 9 & 12 & \\
\hline \multicolumn{7}{|c|}{$\begin{array}{l}\text { SPC, subsequent primary cancer; LDR-BT, low dose-rate brachytherapy; SCC, squamous cell cancer; OOF, out- } \\
\text { of-field; IF, in-field. }\end{array}$} \\
\hline \multicolumn{7}{|c|}{ † Fibrosarcoma, SCC, Melanoma } \\
\hline \multicolumn{7}{|c|}{ †† Head and neck $(n=3)$, Liver $(n=2)$ and one of each: Brain, Eye melanoma, Esophagus, Pancreas, Kidney, } \\
\hline \multicolumn{7}{|c|}{ Malignant schwannoma (muscle), Sarcoma (hand) } \\
\hline
\end{tabular}

Table 3. Subsequent primary cancers fulfilling the criteria of an radiation-induced subsequent primary cancer. 


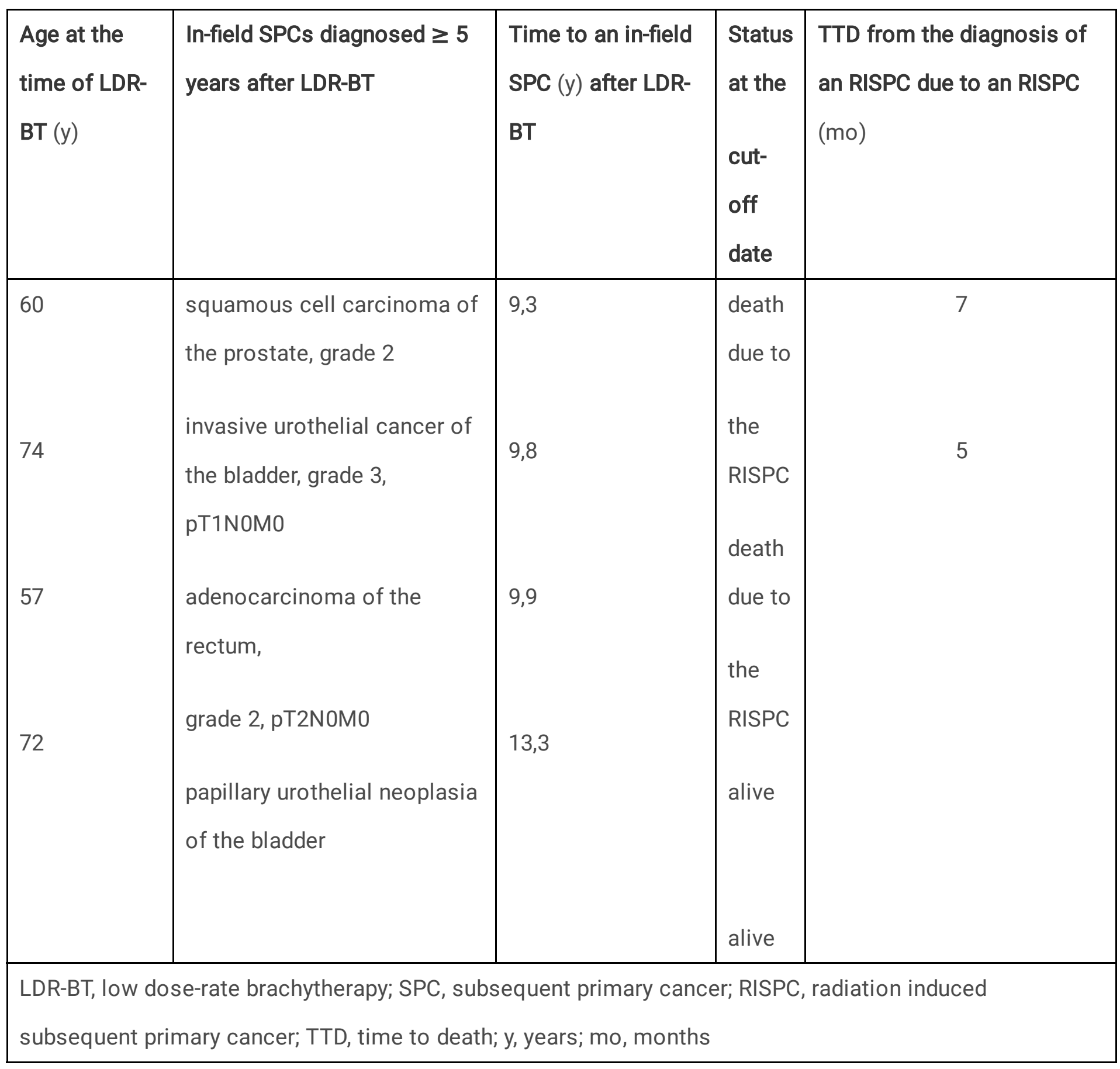

\section{Figures}




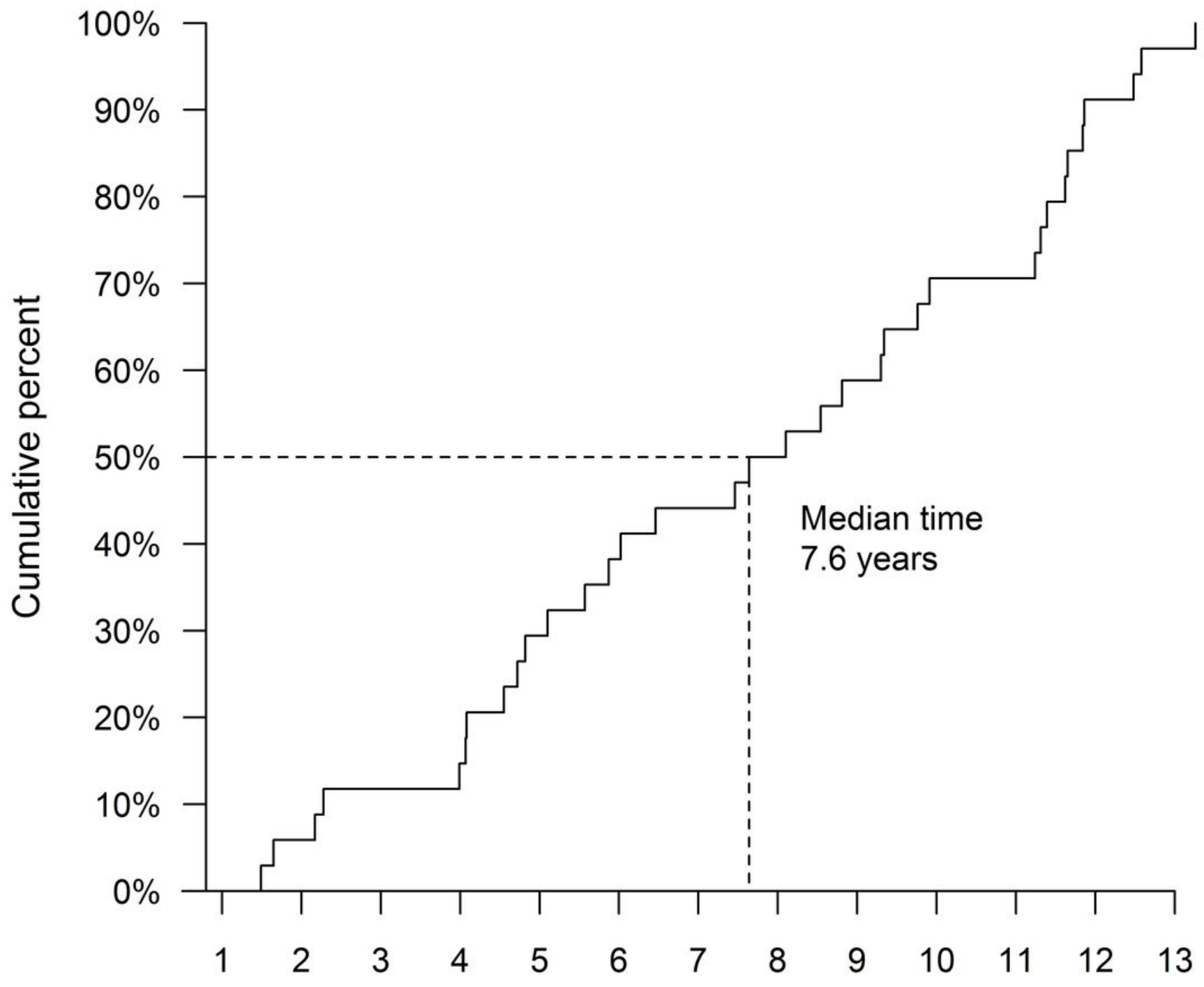

Time, years

Figure 1

The incidence of second primary cancers (SPCs) during the follow-up. 